\title{
Slider Dynamics With Adhesive Force in Near-Field Recording
}

\author{
H. C. Wang, M. S. Huang, and T. S. Liu \\ Department of Mechanical Engineering, National Chiao Tung University, Hsinchu 30010, Taiwan, R.O.C.
}

\begin{abstract}
A near-field optical disk drive uses near-field optics to read/write disk data at a flying height of about $50 \mathrm{~nm}$. Effects of interfacial adhesive forces, including van der Waals forces and electrostatic forces, become more evident during ultralow flying. However, the pickup head-flying-height variation is affected by the suspension, disk flutter, and air flow between the flying head and disk. An analysis model consisting of a simplified suspension and an air-bearing model with springs and dampers in three directions (focusing, pitching, and rolling) is used to simulate the dynamic behavior of the flying head. This study proposes an improved method for measuring the slider's flying-height variation. Simulation results with and without interfacial adhesive forces are compared.
\end{abstract}

Index Terms-Adhesive force, electrostatic force, flying height, near-field recording, van der Waals force.

\section{INTRODUCTION}

D ATA densities of optical storage systems have been growing rapidly in recent years. The near-field optical disk drive (NFODD) is an important development, and its data-storage capacity is substantially larger than DVD optical devices. As the slider flying height is lowered for near-field recording, the adhesive force, including the van der Waals force and electrostatic force between the slider and disk surface, may vary the slider flying height. To deal with the dynamic behavior in near-field recording, we must take into account the adhesive force. In research on pickup head dynamics, the air bearing is described as a spring-damper system [1]-[3]. The influence of the adhesive force in hard-disk recording was presented [4].

This study proposes a dynamic model for near-field recording with adhesive force. Modeling results are compared with experiments.

\section{Discrete Model With AdHEsive Force}

Fig. 1 shows a lumped model of a read-write head system. The slider vertical displacement, pitching angle, and rolling angle are $z_{s}, \theta_{s}$, and $\beta_{s}$, while the disk's vertical displacement, rotation in pitching direction, and rotation in rolling direction are prescribed as $\mathrm{z}_{d}, \theta_{d}$, and $\beta_{d}$, respectively. The suspension and the air bearing are modeled as spring and damper. The spring stiffness in three directions are prescribed as $K_{p}, K_{\theta p}$, and $K_{\beta p}$, while the damping coefficients are prescribed as $C_{p}, C_{\theta p}$, and $C_{\beta p}$, respectively. The spring stiffness of the air bearing in three directions is $K_{A}, K_{\theta A}$, and $K_{\beta A}$, while the damping coefficients of the air bearing are prescribed as $C_{A}$, $C_{\theta A}$, and $C_{\beta A}$. In this study, the total air-bearing forces are treated as concentrated forces acting at B in Fig. 2 to represent nonuniform pressure distribution on the slider. The pitch and

Digital Object Identifier 10.1109/TMAG.2006.888416

Color versions of one or more of the figures in this paper are available online at http://ieeexplore.ieee.org.

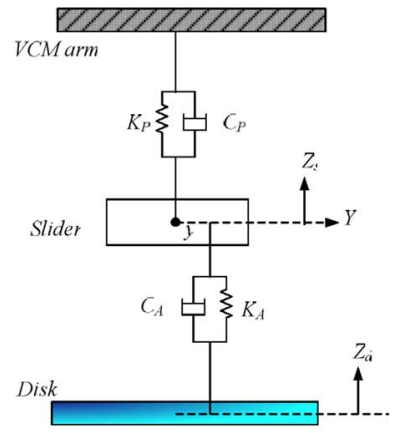

Fig. 1. Lumped model of read-write head system.

roll of the slider are caused by nonequilibrium distribution forces of the air bearing. The symbols $x$ and $y$ are prescribed as the direction from the $X$ and $Y$ axes, respectively.

As the sum of the electrostatic force

$$
\mathrm{F}_{\text {ele }}=A_{n}\left(\frac{\varepsilon_{0} \mathrm{U}^{2}}{2}\right)\left(\frac{1}{\mathrm{~h}^{2}}\right)
$$

and the van der Waals force

$$
\mathrm{F}_{\mathrm{vdw}}=A_{n}\left(\frac{1}{6 \pi}\right)\left(\frac{\mathrm{H}}{\mathrm{h}^{3}}\right)
$$

the adhesive force $F_{a d}$ is shown in the flying-height direction in Fig. 3, where $A_{n}$ is the nominal area, $\varepsilon_{0}$ is permittivity of medium, $U$ is potential difference between two material surfaces, $h$ is the minimum separation between two surfaces, and $\mathrm{H}$ is the Hamaker constant.

Fig. 3 depicts free-body diagrams of the pickup head in three directions. The suspension force consists of a spring $K_{P}$ and a damper $C_{P}$ in Fig. 3. Accounting for the air-bearing effect and adhesive force, a spring-damper force due to air bearing and adhesive force are exerted on the pickup head. According to Newton's law, the equation of motion based on Fig. 3 is given as

$$
\begin{aligned}
m \ddot{z}_{s}+\left(C_{P}+C_{A}\right) \dot{z}_{s}+ & \left(K_{P}+K_{A}\right) z_{s} \\
& =K_{A} z_{d}+C_{A} \dot{z}_{d}-F_{\text {ad }}-P
\end{aligned}
$$




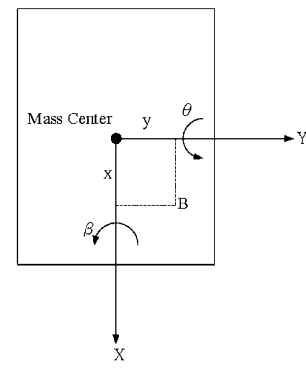

Fig. 2. Air-bearing force acting at point B.

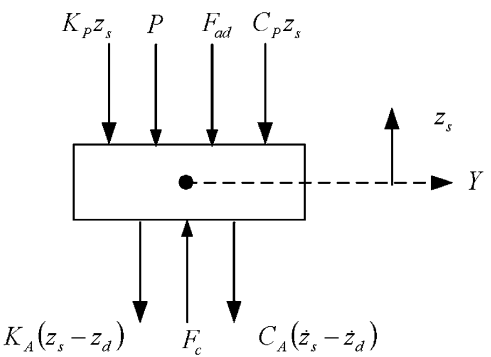

Fig. 3. Free-body diagram of pickup head in focusing direction.

where $P$ is preload acting at the mass center and $F_{\text {ad }}$ denotes the adhesive force. In a similar manner, the equations of motion along the pitching and rolling direction are given as

$$
\begin{aligned}
I_{\theta} \ddot{\theta}_{s} & +\left(C_{\theta P}+C_{\theta A}\right) \dot{\theta}_{s} \\
& +\left(K_{\theta P}+K_{\theta A}\right) \theta_{s}-x K_{A} z_{s}-x C_{A} \dot{z}_{s} \\
& =K_{\theta A} \theta_{d}+C_{\theta A} \dot{\theta}_{d}-x\left(K_{A} z_{d}+C_{A} \dot{z}_{d}\right) \\
I_{\beta} \ddot{\beta}_{s} & +\left(C_{\beta P}+C_{\beta A}\right) \dot{\beta}_{s} \\
+ & \left(K_{\beta P}+K_{\beta A}\right) \beta_{s}+y K_{A} z_{s}+y C_{A} \dot{z}_{s} \\
& =K_{\beta A} \beta_{d}+C_{\beta A} \dot{\beta}_{d}+y\left(K_{A} z_{d}+C_{A} \dot{z}_{d}\right) .
\end{aligned}
$$

According to (3)-(5), the new dynamic equation is written as

$$
\begin{aligned}
& {\left[\begin{array}{ccc}
m & 0 & 0 \\
0 & I_{\beta} & 0 \\
0 & 0 & I_{\theta}
\end{array}\right]\left\{\begin{array}{l}
\ddot{z}_{s} \\
\ddot{\beta}_{s} \\
\ddot{\theta}_{s}
\end{array}\right\}} \\
& +\left[\begin{array}{ccc}
\left(C_{P}+C_{A}\right) & 0 & 0 \\
y C_{A} & \left(C_{\beta P}+C_{\beta A}\right) & 0 \\
-x C_{A} & 0 & \left(C_{\theta P}+C_{\theta A}\right)
\end{array}\right] \\
& \times\left\{\begin{array}{c}
\dot{z}_{s} \\
\dot{\beta}_{s} \\
\dot{\theta}_{s}
\end{array}\right\} \\
& +\left[\begin{array}{ccc}
\left(K_{P}+K_{A}\right) & 0 & 0 \\
y K_{A} & \left(K_{\beta P}+K_{\beta A}\right) & 0 \\
-x K_{A} & 0 & \left(K_{\theta P}+K_{\theta A}\right)
\end{array}\right] \\
& \times\left\{\begin{array}{l}
z_{s} \\
\beta_{s} \\
\theta_{s}
\end{array}\right\} \\
& =\left\{\begin{array}{c}
K_{A} z_{d}+C_{A} \dot{z}_{d}-F_{\mathrm{ad}}-P \\
K_{\beta A} \beta_{d}+C_{\beta A} \dot{\beta}_{d}+y\left(K_{A} z_{d}+C_{A} \dot{z}_{d}\right) \\
K_{\theta A} \theta_{d}+C_{\theta A} \dot{\theta}_{d}-x\left(K_{A} z_{d}+C_{A} \dot{z}_{d}\right)
\end{array}\right\} .
\end{aligned}
$$

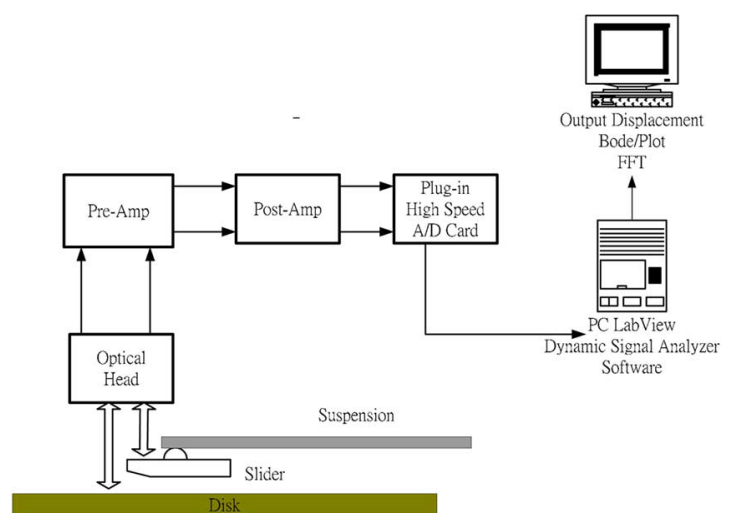

Fig. 4. Experimental setup.
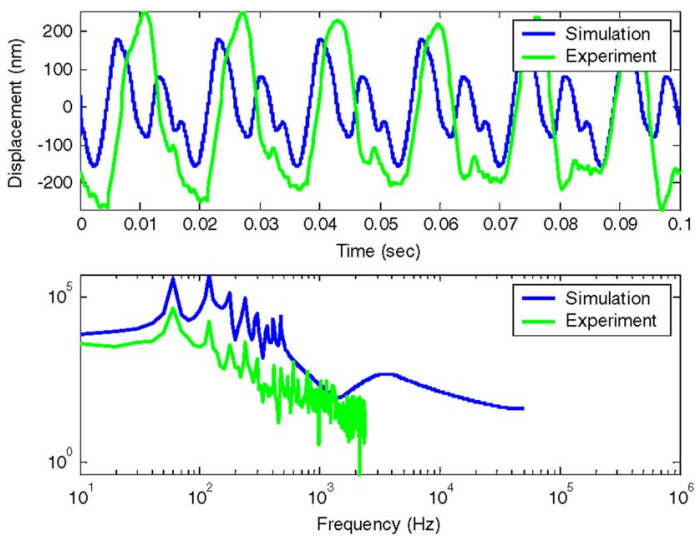

Fig. 5. Flying-height comparison between simulation and experimental results at $3600 \mathrm{rpm}$.

\section{SiMULATION AND EXPERIMENT}

This study uses a laser Doppler vibrometer in measurement as depicted in Fig. 4, where the optical head can generate either a single-beam or dual-beam laser. In the latter, the difference of displacements on both the slider and disk represents the slider flying height. Based on the experimental setup depicted in Fig. 4, the disk vibration was measured and used as disk input data in simulation. Fig. 5 shows that the slider vibration amplitude at $3600 \mathrm{rpm}$ in simulation is $160 \mathrm{~nm}$, while the slider vibration amplitude in the experiment is $220 \mathrm{~nm}$. The fast Fourier transform (FFT) results in Fig. 5 show that the simulation results and experimental results have the same natural frequencies. The first resonance occurs at $60 \mathrm{~Hz}$, which results from disk speed at $3600 \mathrm{rpm}$. In addition, the slider amplitude at $2400 \mathrm{rpm}$ in simulation is $150 \mathrm{~nm}$, while that in the experiment is $210 \mathrm{~nm}$. Accordingly, the faster disk speed results in larger slider vibration in simulation and experiments. The vibration amplitude is smaller in simulation than in experimental results, since spring constants used in simulation are too large.

Fig. 6 shows the FFT results of slider vibrations along the flying-height, pitching, and rolling directions at disk speeds of 3600 and $2400 \mathrm{rpm}$, respectively. The first peaks occur at 60 and $40 \mathrm{~Hz}$, which correspond to disk speeds, respectively. In the flying-height direction, faster disk speed excites more resonances than slower disk speed. The resonances caused by slider pitching are more severe than the resonances caused by slider 


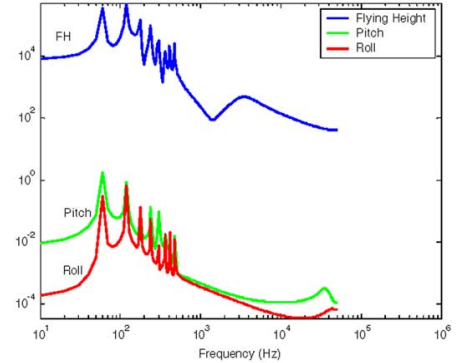

(a)

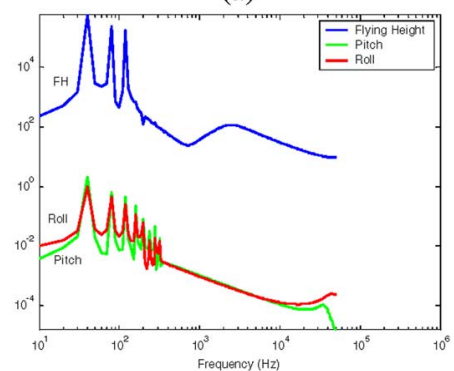

(b)

Fig. 6. Frequency spectrums of slider at (a) 3600 and (b) $2400 \mathrm{rpm}$.

rolling. Hence, pitching vibration is more severe than rolling vibration.

Fig. 7 shows the results calculated by subtracting the flying-height amplitude with adhesive force from the flying-height amplitude without adhesive force. The lower the stable flying height, the larger the flying-height variation caused by the adhesive force. Moreover, the maximum adhesive force at each stable flying height is shown in Fig. 8. When the slider flies lower to a stable flying height at $350 \mathrm{~nm}$, the maximum adhesive force is $95 \mu \mathrm{N}$ that results in a maximum flying-height reduction of $24 \mathrm{~nm}$. Hence, the existence of adhesive force, indeed, influences the slider flying height when a slider flies very close to the disk.

\section{CONCLUSION}

With increasing data-storage capacity, the slider flies above the disk at such a low flying height. To model the slider dynamic behavior, this study proposes a dynamic model incorporating the adhesive force. Computational and experimental results show that the model can account for the dynamic behavior well in near-field recording. The resonances caused by slider pitching are more severe than the resonances caused by slider rolling.

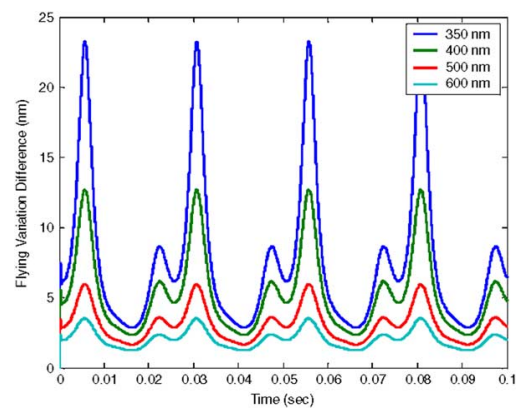

Fig. 7. Flying-height difference among different stable flying heights between a model with adhesive force and a model without adhesive force.

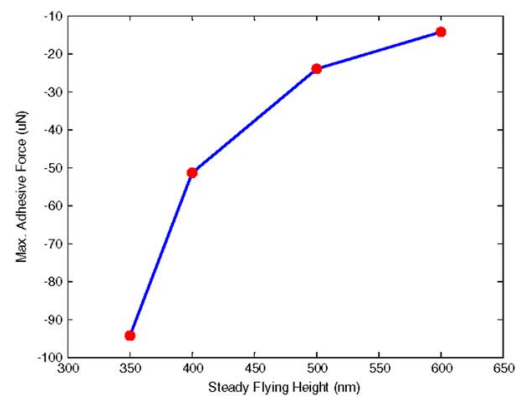

Fig. 8. Relations between adhesive force and stable flying height.

Hence, the van der Waals force and electrostatic force, indeed, affect the slider flying height within the near field.

\section{ACKNOWLEDGMENT}

This work was supported by the "Photonics Sciences and Technology for Tera Era," Center of Excellence, National Science Council, Taiwan, R.O.C., under Grant 94-E-009-009-PAE.

\section{REFERENCES}

[1] K. Ono, J. S. Chen, and D. B. Bogy, "Stability analysis for the head-disk interface in a flexible disk drive," ASME J. Appl. Mech., vol. 58, pp. 1005-1014, 1991.

[2] Q. H. Zeng and D. B. Bogy, "Stiffness and damping evaluation of air bearing sliders and new designs with high damping," ASME J. Tribol., vol. 121, no. 2, pp. 341-347, 1999.

[3] S. Kim, J. Park, and G. Park, "An optical flying head assembly for a small-form-factor plastic disk in PCMCIA-like drive," Jpn. J. Appl. Phys., vol. 43, no. 7B, pp. 4752-4758, 2004.

[4] A. Y. Suh and A. A. Polycarpou, "Adhesive contact modeling for sub-5-nm ultralow flying magnetic storage," J. Appl. Phys., no. 97, pp. 104328-1-140328-11, 2005.

Manuscript received August 10, 2006 (e-mail: reic.me90g@nctu.edu.tw). 